\title{
EDITORIAL
}

\section{Introduction to special issue on cellular vehicles}

Gene Therapy (2008) 15, 703; doi:10.1038/gt.2008.46; published online 27 March 2008

A famous Russian philosopher, Mikhail Sholokhov, once noted that it is nearly impossible to understand history when you are living in the middle of it.' Such is also the fact with the birth of a new field of endeavor. In this regard, the past couple of years have seen the emergence of a new science that seeks to pair gene therapy and cellular therapy for therapeutic intent. Indeed, there has been a virtual explosion of the number of 'cellular vehicle' approaches being developed and applied toward the realization of novel targeted therapeutics.

Strategies that pair cellular therapy and gene therapy for the achievement of therapeutic gene delivery provoke many of the same methodological issues as embodied in the parental technologies. But cellular vehicle approaches also raise distinct issues based on the unique pairing of methods. Indeed, these emerging reports based on cellular vehicles represent the leading edge of a new field of scientific endeavor.

The current Special Issue of Gene Therapy is devoted to this emerging discipline. There are a range of reports that embody the full spectrum and promise of this technology. These reports also highlight the novel technical, strategic and regulatory issues that will be provoked as these cellular vehicle strategies advance from proof of principle to human clinical translation. Indeed, the rapid advancement of this field has truly outstripped the means by which such key technical and regulatory aspects have been addressed.

Nonetheless, we have been fortunate to engage in this effort the leaders of this new field and to bear witness to the novel paradigms they present. The apparent diffuseness of themes is belied by the fact that all these reports are based on the central science of cellular vehicles. And thereby this Special Issue seeks to provide that most fleeting of possibilities-to understand history while living in the middle of it.

D Curiel

Division of Human Gene Therapy, The Gene Therapy Center, University of Alabama at Birmingham, Birmingham, AL, USA E-mail: curiel@uab.edu 